\title{
Static Analysis of FG Beams via Complementary Functions Method
}

\author{
Ahmad Reshad Noori ${ }^{1}$ (D), Timuçin Alp Aslan ${ }^{2 *}$ (D) Beytullah Temel ${ }^{2}$ \\ 'Istanbul Gelisim University, Department of Civil Engineering, Istanbul, Turkey \\ ${ }^{2}$ Cukurova University, Department of Civil Engineering, Adana, Turkey
}

\begin{abstract}
The bending response of Functionally Graded (FG) beams is carried out by the Complementary Functions Method (CFM). The mechanical properties of the material, Young's modulus, of the straight beams are considered to be graded only in the thickness direction. Poisson's ratio is supposed to be constant. Governing equations of the considered problem are obtained with the aid of minimum total potential energy principle based on Timoshenko's beam theory (FSDT). The main purpose of this paper is the infusion of the CFM to the bending analysis of FG straight beams. The effectiveness and accuracy of the proposed scheme are confirmed by comparing its numerical results with those of the available literature. The application of this efficient method provides accurate results of static response for FGM beams with different variations of material properties.
\end{abstract}

Keywords: FGM beams; Complementary Functions Method (CFM); Static Analysis; Shear Deformation.

\section{INTRODUCTION}

FGM beams are extensively applied in many practical applications of engineering. Moreover, straight beams made by using FG materials are mainly used in the construction of modern engineering structures. Because of their extensive usage, there are many papers dealing with the static and dynamic analysis of the FG beams. Several numerical and analytical methods are applied by engineers and scientists in order to investigate the behavior of those important structures. Some of those studies are summarized as follows:

A shear deformable finite element (FE) was developed by [1] for static and dynamic analysis of beams with FGM layers. They considered FSDT in their study. [2] presented an analytical solution for a cantilever FG beam. Solutions are obtained for several loading cases. They assumed the variation of the material properties in the thickness direction of the beam. [3] carried out the FE for static equilibrium equations of FG beams. They obtained two stiffness matrices by considering the influence of rotation and shear deformation. [4] presented a unified approach to study the static and dynamic analysis of FG beams by including the effects of shear deformation. They extended the Timoshenko beam theory to study FG beams.

The static response of FG beams was examined by [5] with the aid of the Ritz method. The beams were considered that their material is FG through the thickness direction. [6] derived the governing equation for static and dynamic responses of FG beams by considering the influence of shear deformation. They extended the Levinson beam theory to FG beams. A refined shear deformation theory was developed by [7] for the static and dynamic analysis of FG beams. Their developed theory doesn't need any shear correction factor.

Several deformation theories for static and dynamic response of FG beams were presented by [8]. They also examined the effects of the material gradient index on the bending behavior of the considered structures. A refined exponential shear deformation theory was suggested by [9] to study the bending behavior of FG beams. [10] developed an analytical relationship between the static response of Euler Bernoulli and Timoshenko FG beams. They investigated the effects of material inhomogeneity and shear deformation on the static behavior of FG beams. An efficient approach was suggested by [11] to study the static response of FG beams with the aid of the finite volume method and Timoshenko's beam theory. [12] presented a one-dimensional FE based on zig-zag theory to carry out the static behavior and natural frequencies of FG beams. They used linear interpolation for axial displacements and deflections. A FE model was presented by [13] to examine the behavior of homogenous and FG beams. Shear deformation was considered in their formulations. They built a FORTRAN code to predict the static response. The
* Corresponding authour

Email: taslan@cu.edu.tr
European Mechanical Science, March 2020, 4(1): 1-6 doi: https://doi.org/10.26701/ems.590864

Received: July 11, 2019

Accepted: January 24, 2020 
CFM is successfully applied previously in structural mechanics problems by [14-20]

The main aim of this study is to suggest an accurate and effective numerical approach for the bending analysis of FG straight beams. The mechanical properties of the material are assumed to vary continuously in the thickness direction of the beam according to a power law form. We studied the static response of FG straight beams made of metal alloy and ceramic for several boundary conditions. The influence of the material properties on the static response of the considered beams is carried out. The equations which govern the static response of the considered structure are obtained with the aid of minimum total potential energy based on the Timoshenko's beam theory. For numerical calculations, the fifth-order Runge -Kutta (RK5) algorithm is applied. To predict the static response of FG beams by the presented procedure a computer program is prepared in Fortran. To demonstrate the validity and applicability of the current approach, some of the present results are compared with the results of the literature. Accuracy and good agreement are observed.

\section{MATERIAL AND THEORY}

\subsection{Functionally Graded Materials}

Consider a FG straight beam as given in Figure 1. The Young's modulus of this beam is considered to vary continuously only in the thickness direction of the beam by Eq. (1). The Poisson's ratio $(v)$ is considered to be constant.

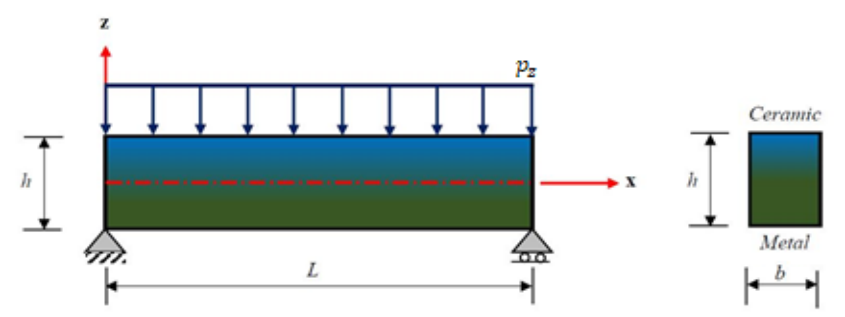

Figure 1. FG beam

$$
E(z)=\left(E_{t}-E_{b}\right)\left(\frac{z}{h}+\frac{1}{2}\right)^{n_{z}}+E_{b}
$$

where, $E_{t}$ and $E_{b}$ are the modulus of elasticity of the top and bottom of the beam. $n_{z}$ is a constant defining the variation of modulus of elasticity along the thickness direction of the considered beam. It can be clearly seen that when $z=-h / 2$ the $E(z)=E_{b}$ and $z=+h / 2$ the $E(z)=E_{t}$. When the $n_{z}=0$ the material of the beam is isotropic homogeneous material.

\subsection{Governing Equations}

The displacement field for the considered beam based on FSDT can be given as follows:

$$
\begin{aligned}
& U_{x}=u(x)+z \theta(x) \\
& U_{z}=w(x)
\end{aligned}
$$

where $u$ is the axial displacement, $w$ is the vertical deflection and $\theta$ is the rotating angle along the mid-plane of the beam $(z=0)$. The relationship between the displacements and strains can be given by Eqs. (4-5).

$$
\begin{aligned}
& \varepsilon_{x}=\frac{\partial U_{x}}{\partial x}=u^{\prime}+z \theta^{\prime} \\
& \gamma_{x, y}=\theta+w^{\prime}
\end{aligned}
$$

The relations of stress-strain for the considered FG straight beam are:

$$
\begin{aligned}
& \sigma_{x}=E(z) \varepsilon_{x} \\
& \tau_{x z}=G(z) \gamma_{x z}=\frac{E(z)}{2(1+v)} \gamma_{x z}
\end{aligned}
$$

where $G(z)$ is the shear modulus. The relationships between internal forces(axial force $N_{x}$, moment of the bending $M_{x}$, and shear force $Q_{z}$ ) and stresses are given in the integral form as follows.

$$
\begin{aligned}
& N_{x}=b \int_{-h / 2}^{+h / 2} \sigma_{x} d z=b \int_{-h / 2}^{+h / 2} E(z) \varepsilon_{x} d z \\
& M_{x}=b \int_{-h / 2}^{+h / 2} \sigma_{x} z d z=b \int_{-h / 2}^{+h / 2} E(z) \varepsilon_{x} z d z \\
& Q_{z}=b \int_{-h / 2}^{+h / 2} k_{s} \tau_{x z} d z=b \int_{-h / 2}^{+h / 2} k_{s} \frac{E(z)}{2(1+v)} \gamma_{x z} d z
\end{aligned}
$$

where $k_{s}$ is the shear correction factor. Constitutive equations of the considered beam can be given as follows:

$$
\begin{aligned}
& N_{x}=A_{11} u^{\prime}+A_{12} \theta^{\prime} \\
& Q_{z}=A_{33}\left(\theta+w^{\prime}\right) \\
& M_{x}=A_{12} u^{\prime}+A_{22} \theta^{\prime}
\end{aligned}
$$

where

$$
\begin{aligned}
& A_{11}=b \int_{-h / 2}^{+h / 2} E(z) d z \\
& A_{12}=b \int_{-h / 2}^{+h / 2} E(z) d z \\
& A_{22}=b \int_{-h / 2}^{+h / 2} E(z) z^{2} d z \\
& A_{33}=k_{s} b \int_{-h / 2}^{+h / 2} \frac{E(z)}{2(1+v)} d z
\end{aligned}
$$

The minimum total potential energy principle is used to obtain the governing equations of the FG beams as follows:

$$
\Pi_{t}=\int_{0}^{L} \underbrace{\int_{A} \frac{1}{2}\left(\sigma_{x} \varepsilon_{x}+\tau_{x z} \gamma_{x z}\right)-\left(p_{x} u+p_{z} w\right) d A}_{l} d x
$$

The minimum total potential energy is

$$
\delta \Pi_{t}=\delta \int_{0}^{L} l d x=0
$$

By defining the canonically conjugate momentums and their derivatives to the coordinates $(u, w, \theta)$ the set of canonical ordinary differential equations can be obtained as follows:

$$
\begin{aligned}
& \frac{d u}{d x}=\frac{A_{12} M_{x}-A_{22} N_{x}}{b\left(A_{12}{ }^{2}-A_{11} A_{22}\right)} \\
& \frac{d w}{d x}=\frac{Q_{z}}{b k_{s} A_{33}}-\theta \\
& \frac{d \theta}{d x}=\frac{-A_{11} M_{x}+A_{12} N_{x}}{b\left(A_{12}{ }^{2}-A_{11} A_{22}\right)} \\
& \frac{d N_{x}}{d x}=-p_{x}
\end{aligned}
$$




$$
\begin{aligned}
\frac{d Q_{z}}{d x} & =-p_{z} \\
\frac{d M_{z}}{d x} & =Q_{z}
\end{aligned}
$$

The bending response of the considered beam is examined using the CFM. The RK5 algorithm is used to carry out the initial value problem based on the CFM. The obtained first order 6 sets of ordinary differential equations can be expressed in matrix form as follows.

$$
\frac{d\{\mathbf{Y}(x)\}}{d x}=[\mathbf{A}(x)]\{\mathbf{Y}(x)\}+\{\mathbf{F}(x)\}
$$

For the solution of the initial-value problem based on the CFM see [14]. Using this method does not calculate only the unknown function itself (displacement) but also calculates its derivatives (rotations, internal forces and moments). This method can be applied for any arbitrary continues functions. To obtain the general solution of the governing equation, boundary conditions are required. Boundary conditions are as follows:

\section{Simply supported beam (S-S)}

$$
\begin{aligned}
& u=0 ; \quad w=0 ; \quad M_{x}=0 \quad \text { at } x=0 \\
& w=0 ; \quad N_{x}=0 ; \quad M_{x}=0 \quad \text { at } \quad x=L
\end{aligned}
$$

\section{Cantilever beam $(C)$}

$$
\begin{aligned}
& u=0 ; \quad w=0 ; \quad \theta=0 \quad \text { at } x=0 \\
& N_{x}=0 ; \quad Q_{z}=0 ; \quad M_{x}=0 \text { at } x=L
\end{aligned}
$$

\section{Clamped-clamped supported beam (C-C)}

$$
\begin{aligned}
& u=0 ; \quad w=0 ; \quad \theta=0 \text { at } x=0 \\
& u=0 ; \quad w=0 ; \quad \theta=0 \text { at } x=L
\end{aligned}
$$

\section{NUMERICAL EXAMPLES AND DISCUSSION}

In this study, a computer program is coded in Fortran programing language which examines the bending analysis of FG beams. The static response of FG beams is investigated for various boundary conditions and several material gradient indices. In this section, numerical results are carried out to verify the accuracy of the presented approach and to investigate the displacements of FG beams. As the shear correction factor, a value of $k_{s}=5 / 6$ is used. To obtain non-dimensional vertical displacements of the considered structure under the uniformly distributed load, the following terms are used.

$$
\begin{array}{ll}
\bar{w}=w\left(\frac{32 E_{A l} b h^{3}}{5 p_{z} L^{4}}\right) & \text { for simply supported beam } \\
\bar{w}=w\left(\frac{2 E_{A l} b h^{3}}{3 p_{z} L^{4}}\right) & \text { for cantilever beam } \\
\bar{w}=w\left(\frac{32 E_{A l} b h^{3}}{5 p_{z} L^{4}}\right) & \text { for clamped-clamped beam }
\end{array}
$$

\begin{tabular}{|c|c|c|c|c|c|c|c|}
\hline \multirow{2}{*}{$\mathrm{L} / \mathrm{h}$} & \multirow{2}{*}{ References } & \multicolumn{6}{|c|}{ Material Gradient Index, $n_{z}$} \\
\hline & & Full Metal & 0.5 & 1 & 2 & 5 & Full Ceramic \\
\hline \multirow{6}{*}{4} & Ref. [5] $\left(k_{s}=1\right)$ & 1.13002 & 0.71482 & 0.62936 & 0.56165 & 0.49176 & 0.39550 \\
\hline & Ref. $[21]\left(k_{s}=1\right)$ & 1.13000 & 0.71472 & 0.62933 & 0.56163 & 0.49175 & 0.39550 \\
\hline & Ref. [21] $\left(k_{s}=5 / 6\right)$ & 1.15600 & 0.73078 & 0.64281 & 0.57325 & 0.50196 & 0.40460 \\
\hline & Present Study $\left(k_{s}=1\right)$ & 1.13000 & 0.71517 & 0.62935 & 0.56164 & 0.49176 & 0.39550 \\
\hline & Present Study $\left(k_{s}=5 / 6\right)$ & 1.15600 & 0.73123 & 0.64283 & 0.57326 & 0.50196 & 0.40460 \\
\hline & Relative Error $\left(k_{s}=5 / 6\right)$ & $3.24 \mathrm{E}-08$ & $6.17 \mathrm{E}-04$ & $3.35 \mathrm{E}-05$ & $1.87 \mathrm{E}-05$ & 4.20E-06 & $2.74 \mathrm{E}-16$ \\
\hline \multirow{6}{*}{16} & Ref. [5] $\left(k_{s}=1\right)$ & 1.00812 & 0.63953 & 0.56615 & 0.50718 & 0.44391 & 0.35284 \\
\hline & Ref. $[21]\left(k_{s}=1\right)$ & 1.00810 & 0.63944 & 0.56613 & 0.50717 & 0.44391 & 0.35284 \\
\hline & Ref. [21] $\left(k_{s}=5 / 6\right)$ & 1.00980 & 0.64045 & 0.56698 & 0.50790 & 0.44455 & 0.35341 \\
\hline & Present Study $\left(k_{s}=1\right)$ & 1.00813 & 0.63989 & 0.56616 & 0.50719 & 0.44392 & 0.35284 \\
\hline & Present Study $\left(k_{s}=5 / 6\right)$ & 1.00975 & 0.64090 & 0.56700 & 0.50791 & 0.44456 & 0.35341 \\
\hline & Relative Error $\left(k_{s}=5 / 6\right)$ & 4.95E-05 & $6.98 \mathrm{E}-04$ & $3.19 \mathrm{E}-05$ & $2.94 \mathrm{E}-05$ & $1.24 \mathrm{E}-05$ & 7.07E-06 \\
\hline
\end{tabular}

\begin{tabular}{|c|c|c|c|c|c|c|}
\hline \multirow{2}{*}{$\mathrm{L} / \mathrm{h}$} & \multirow{2}{*}{ Theory } & \multicolumn{5}{|c|}{ Material Gradient Index, $n_{z}$} \\
\hline & & Full Ceramic & 1 & 2 & 5 & Full Metal \\
\hline \multirow{6}{*}{4} & \multicolumn{6}{|l|}{ S-S Beam } \\
\hline & FSDT & 0.40460 & 0.64283 & 0.73519 & 0.82403 & 1.15600 \\
\hline & \multicolumn{6}{|l|}{ C Beam } \\
\hline & FSDT & 0.37275 & 0.59565 & 0.67899 & 0.75454 & 1.06500 \\
\hline & \multicolumn{6}{|l|}{ C-C Beam } \\
\hline & FSDT & 0.62300 & 0.96639 & 1.12061 & 1.30054 & 1.78000 \\
\hline \multirow{6}{*}{16} & \multicolumn{6}{|l|}{ S-S Beam } \\
\hline & FSDT & 0.35341 & 0.56700 & 0.64486 & 0.71235 & 1.00975 \\
\hline & \multicolumn{6}{|l|}{ C Beam } \\
\hline & FSDT & 0.35142 & 0.56405 & 0.64135 & 0.70801 & 1.00407 \\
\hline & \multicolumn{6}{|l|}{ C-C Beam } \\
\hline & FSDT & 0.36706 & 0.58722 & 0.66895 & 0.74213 & 1.04875 \\
\hline
\end{tabular}
where $p_{z}$ is uniformly distributed load (see Figure 1), $E_{A l}$ is Young's modulus of Aluminum, is the vertical displacement $(z=0), L$ is the beam length, $b$ is the width and $h$ is the height of the beam cross-section. When the literature is reviewed it can be seen that Eq. (33) has been published incorrectly in

Table 1. Non-dimensional maximum vertical displacements of S-S FG beams subjected a uniformly distributed load.

Table 2. Non-dimensional maximum vertical displacements of FG beams subjected a uniformly distributed load. 
[5] due to some typos errors.

Firstly, the static responses of two S-S FG beams with $L / h$ ratios of 4 and 16 under uniform load are investigated. FGMs are composed of Aluminum (Al: $E_{t}=E_{A l}=70 \mathrm{GPa} ; v_{A l}=0.3$ ) in the top surface and Zirconia $\left(\mathrm{ZnO}_{2}: E_{b}=E_{z r}=200 \mathrm{GPa}\right.$; $\left.v_{z r}=0.3\right)$ in the bottom surface. To confirm the presented results, comparisons were made with those of [5] and [21]. The solutions are calculated at 11 collocation points through the length of the beam (10 divisions). The maximum dimensionless vertical displacements for various values of material gradient index are tabulated in Table 1. It must be noted, that even though it is reported in [5] that the shear correction has been taken as $k_{s}=5 / 6$ but it must be 1 in [5] so that their reported results can be obtained. It can be seen clearly in Table 1 that current results are in excellent agreement with previous studies. Relative error is defined as $\left|\frac{\mid R e f 211-C F M}{R e f(21]}\right|$ and is calculated for maximum displacements. It can be observed in this table that displacement results decrease with increasing the value of material gradient index. It should be emphasized that as the material gradient index $n_{z}$ increases, the FG beams approach to full ceramic beam.

The maximum non-dimensional vertical displacements for various boundary conditions and several material gradient index are listed in Table 2. Unless expressly provided otherwise, the bottom surface of the beams is always assumed to be Aluminum (Al: $E_{b}=E_{A l}=70 \mathrm{GPa} ; v_{A l}=0.3$ ) and the top surface of the beam is always considered to be Zirconia $\left(\mathrm{ZnO}_{2}: E_{b t}=E_{z r}=200 \mathrm{GPa} ; v_{z r}=0.3\right)$ in the given examples. In sharp contrast to the previous case, in this example, maximum non-dimensional vertical displacements increase as the material gradient index increase (Table 2).

Axial displacement, transverse deflection, and rotation along the axis of the beam are given in Figures. (2-4). All of those quantities increase with increasing the value of the material gradient index. When the beam is homogenous, axial displacement doesn't occur. Heterogeneity of the material causes axial displacement in those FG beams which are only subjected to vertical loads. Figures (2-4) are plotted for a clamped-clamped FG beam, with $L=0.4 \mathrm{~m}, b=0.1 \mathrm{~m}$, $h=0.1 \mathrm{~m}$ subjected to a uniform distributed load of $p_{z}=100$ $\mathrm{N} / \mathrm{m}$.

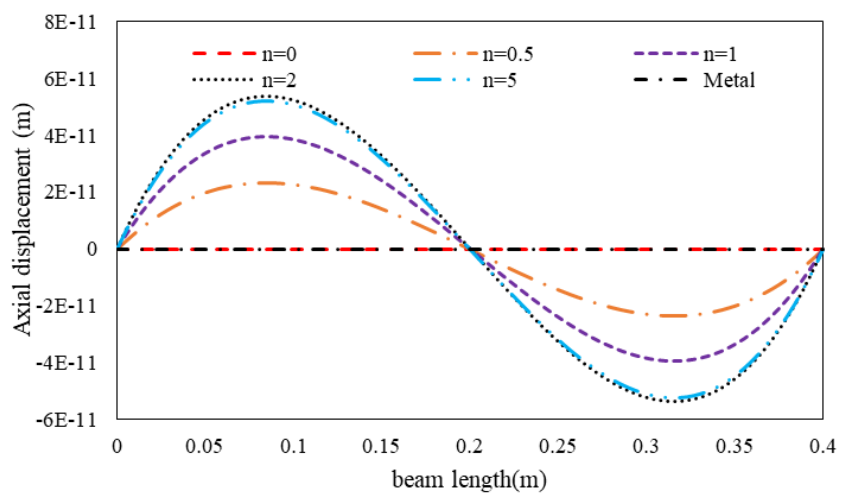

Figure 2. Axial displacement of C-C FG beam.

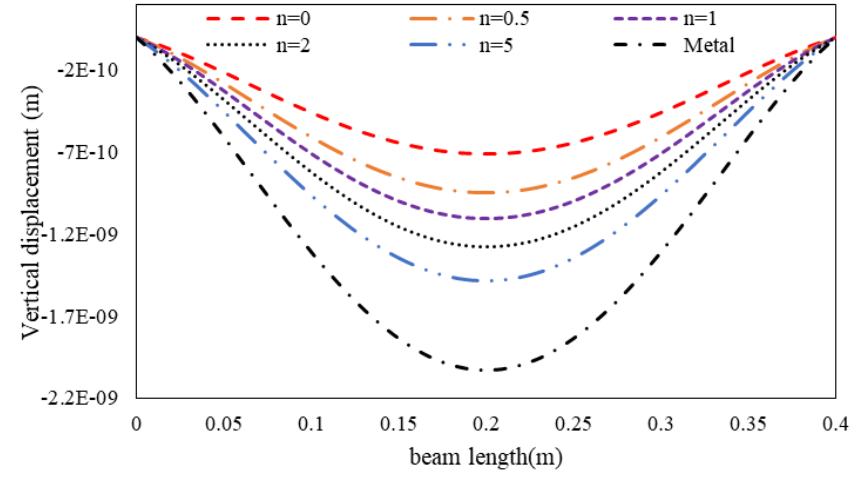

Figure 3. Vertical displacement of C-C FG beam.

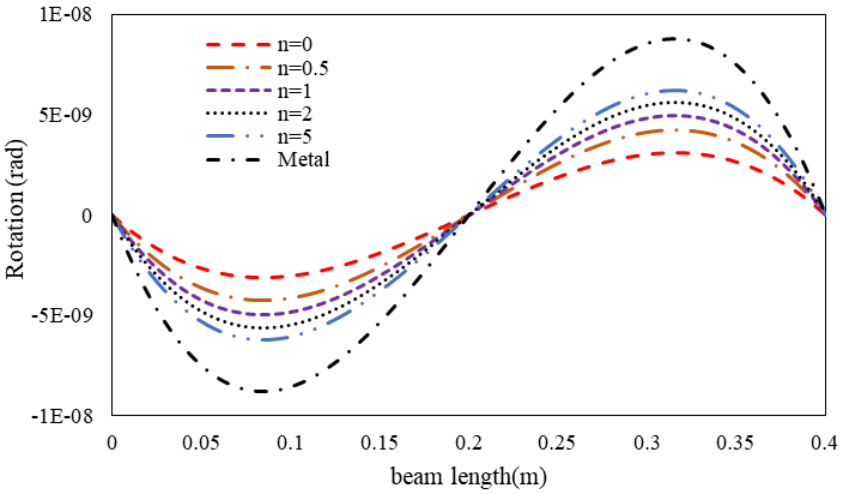

Figure 4. Rotation of C-C FG beam.

The axial and vertical displacements, rotations and bending moment values of FG beams are illustrated in Figures (5-8) for several kinds of support types to show the effect of various boundary conditions on the static response of the present class problem. The geometric properties of the beam and the loading are assumed to be the same as the previous example. The material gradient index, $n_{z}$, is taken to be 1 .

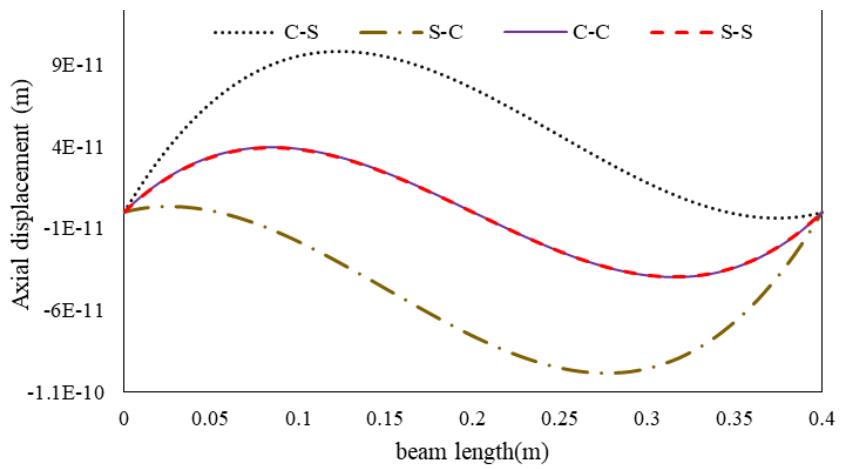

Figure 5. Axial displacement of FG beams.

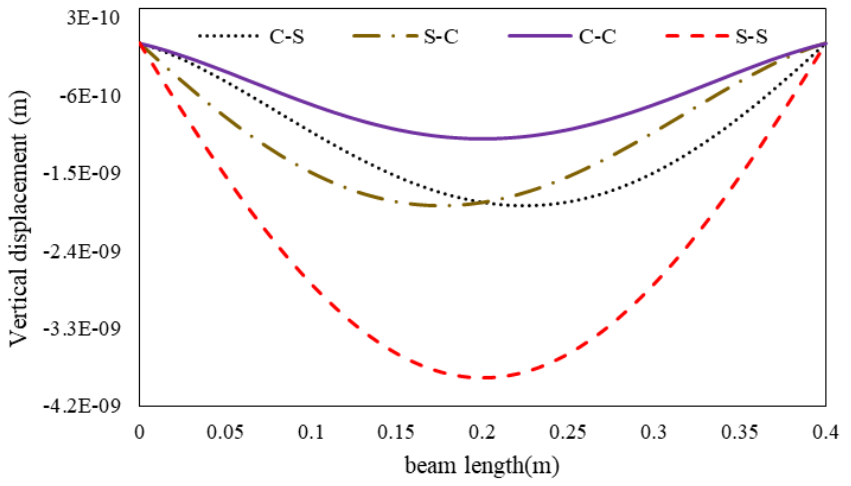

Figure 6. Vertical displacement of FG beams. 


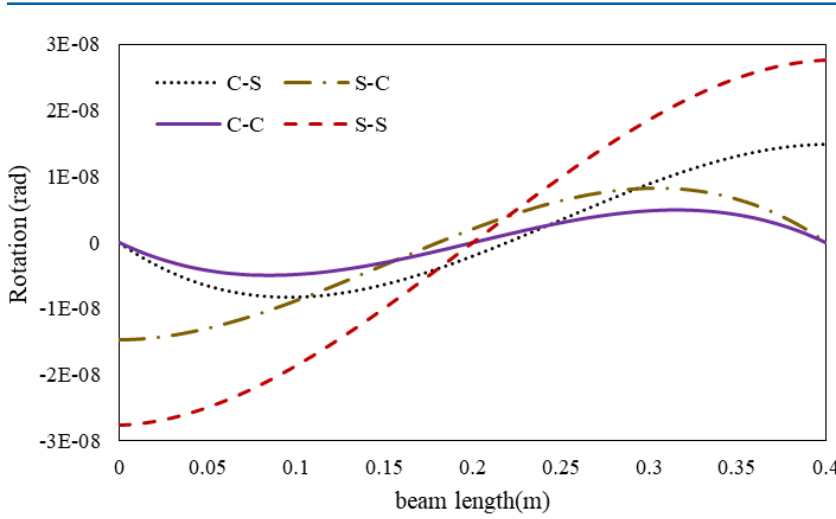

Figure 7. Rotation of FG beams.

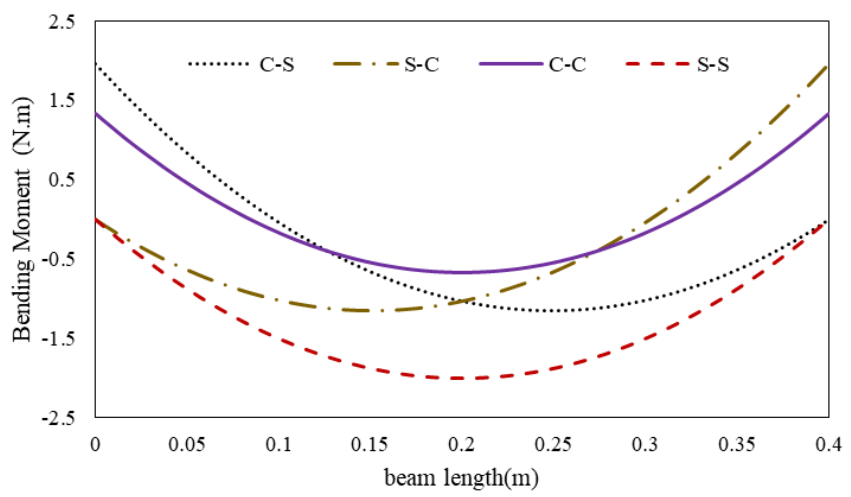

Figure 8. Bending moment of FG beams.

It can be seen in Figure 5, that the axial displacement values of S-S and C-C FG beams are the same. Figure 6-7 demonstrate that $\mathrm{C}-\mathrm{C}$ beam has the smallest vertical deflection and rotation while S-S beam has the highest transverse deflection and rotation. It is clear in Figure 8 that the internal bending moment values are higher in beams with C-S and S-C supports than those of $\mathrm{C}-\mathrm{C}$ supported beams.

\section{CONCLUSION}

The main purpose of this paper is the application of the CFM to static response of FG beams. Governing equations of FG beams are carried out by the principle of minimum total potential energy based on the Timoshenko's beam theory. By applying the current procedure, the static response of FG beams subjected to arbitrary axial and vertical loads can be examined for various boundary conditions. Results of the suggested model have been validated for several types of FG beams. A computer program is coded in Fortran. In the solution process the effect of shear deformation is considered. The results obtained from the program are compared with the results of available literature in tabular form and excellent agreement is observed. Besides, the effects of material gradient index and boundary conditions on the static response of FG beams are investigated in detail. Given tables and Figures evinced that change of the material gradient index has a remarkable influence on the static response of FG beams.

\section{REFERENCES}

[1] Chakraborty A, Gopalakrishnan S, Reddy J, (2003). A new beam finite element for the analysis of functionally graded materials. Inter- national Journal of Mechanical Sciences. 45(3):519-39.

[2] Zhong Z, Yu T, (2007). Analytical solution of a cantilever functionally graded beam. Composites Science and Technology. 67(34):481-8.

[3] Kadoli R, Akhtar K, Ganesan N, (2008). Static analysis of functionally graded beams using higher order shear deformation theory. Applied Mathematical Modelling. 32(12):2509-25.

[4] Li X-F, (2008). A unified approach for analyzing static and dynamic behaviors of functionally graded Timoshenko and Euler-Bernoulli beams. Journal of Sound and Vibration. 318(4-5):1210-29.

[5] Şimşek M, (2009). Static analysis of a functionally graded beam under a uniformly distributed load by Ritz method. International Journal of Engineering \& Applied Sciences. 1(3):1-11.

[6] Li X-F, Wang B-L, Han J-C, (2010). A higher-order theory for static and dynamic analyses of functionally graded beams. Archive of Applied Mechanics. 80(10):1197-212.

[7] Nguyen T-K, Vo TP, Thai H-T, (2013). Static and free vibration of axially loaded functionally graded beams based on the first-order shear deformation theory. Composites Part B: Engineering. 55:147-57.

[8] Thai H-T, Vo TP, (2012). Bending and free vibration of functionally graded beams using various higher-order shear deformation beam theories. International Journal of Mechanical Sciences.62(1):57-66.

[9] Hadji L, Khelifa Z, Daouadji T, Bedia E, (2015). Static bending and free vibration of FGM beam using an exponential shear deformation theory. Coupled Systems Mechanics. 4(1):99-114.

[10] Li S-R, Cao D-F, Wan Z-Q, (2013). Bending solutions of FGM Timoshenko beams from those of the homogenous Euler-Bernoulli beams. Applied Mathematical Modelling. 37(10-11):7077-85.

[11] Jing L-I, Ming P-j, Zhang W-p, Fu L-r, Cao Y-p, (2016). Static and free vibration analysis of functionally graded beams by combination Timoshenko theory and finite volume method. Composite structures. 138:192-213.

[12] Khan AA, Naushad Alam M, Wajid M, (2016). Finite element modelling for static and free vibration response of functionally graded beam. Latin American Journal of Solids and Structures. 13(4):690714.

[13] Ziou H, Guenfoud H, Guenfoud M, (2016). Numerical modelling of a Timoshenko FGM beam using the finite element method. International Journal of Structural engineering. 7(3):239-61.

[14] Temel B, Aslan TA, Noori AR, (2017).An Efficient Dynamic Analysis of Planar Arches, European Mechanical Science. 1(3):82-88.

[15] Temel B, Yildirim S, Tutuncu N, (2014). Elastic and viscoelastic response of heterogeneous annular structures under Arbitrary Transient Pressure. International Journal of Mechanical Sciences. 89:78-83.

[16] Yildirim S, Tutuncu N, (2018). Axisymmetric plane vibration analysis of polar-anisotropic disks. Composite Structures. 194:509-515.

[17] Yildirim S, Tutuncu N, (2018). Radial vibration analysis of heterogeneous and non-uniform disks via complementary functions method. The Journal of Strain Analysis for Engineering Design. 53(5):332-337.

[18] Eker M, Yarımpabuç D, Çelebi K, (2018). The Effect of the Poisson Ratio on Stresses of Heterogeneous Pressure Vessels. European Mechanical Science. 2(2): 52-59.

[19] Yarımpabuç D, Temo A, (2018). The Effect of Uniform Magnetic Field on Pressurized FG Cylindirical and Spherical Vessels. European Mechanical Science. 3(4): 133-141. 
[20] Noori AR, Temel B. On the vibration analysis of laminated composite parabolic arches with variable cross-section of various ply stacking sequences. Mechanics of Advanced Materials and Structures 2018; DOI: 10.1080/15376494.2018.1524949.

[21] Vo TP, Thai H., Nguyen TK, Inam F, (2014). Static and vibration analysis of functionally graded beams using refined shear deformation theory. Meccanica. 49: 155. 